\title{
Visualising Spatial Light Intensity Distribution on Optical Fibre Core with Spreadsheet
}

\author{
Elisabeth Pratidhina*1,20, Heru Kuswanto ${ }^{1}$, Wipsar Sunu Brams Dwandaru ${ }^{1}$ \\ ${ }^{1}$ Physics Education, Postgraduate Program, Universitas Negeri Yogyakarta, Jalan Colombo No 1, 55281, Yogyakarta, \\ Indonesia, \\ ${ }^{2}$ Department of Physics Education, Widya Mandala Catholic University, Jalan Kalijudan 37 Surabaya, Indonesia
}

Received on May 14, 2020. Revised on June 15, 2020. Accepted on June 18, 2020.

\begin{abstract}
Spreadsheet software is a practical and powerful computing and graphical tool. This study presents a simple way to build a physics simulation in spreadsheet software. The simulation aims to visualise spatial light intensity distribution on an optical fibre core. The simulation can visualise various modes. The mathematical equation in optical fibre is quite complicated. The visualisation may help students in interpreting the mathematical equation and making a connection between theoretical modelling and experiment.
\end{abstract}

Keywords: Spreadsheet, Optical Fibre, Mode, Visualisation

\section{Introduction}

Spreadsheet is a powerful computing and graphical tool. Users can use spreadsheet easily without requiring complex programming language. Therefore, spreadsheet is widely used by people who work in various fields such as economy, engineering, and physics.

In physics, people use spreadsheet for research and education. For research purposes, it can be used to analyse the research data and display the data in a more communicative form, such as a chart. For education purposes, we can build simple simulations and visualisations when we are discussing a physical equation.

Integrating simulation in physics teaching has some benefits for students, such as fostering understanding in science content, stimulating inquiry skills [1], and enhancing metamodeling knowledge [2]. Through variables manipulation and physical effect observation in simulation, the learning process becomes more applicable to real-world problems [3]. Moreover, real-experiments that are too complex, costly, time-consuming, or risky to be handled in the classroom are possible to be re-created via simulation [4]. As simulation can simplify the real complex environment, it can reduce cognitive load and allow the students to focus on specific target concepts $[1]$.

Spreadsheet provides a powerful feature that can be utilised for simulating physical phenomena. Because there is no need to use complex programming language, spreadsheet is very appealing for both introductory and advanced physics class. There are several papers which present the application of spreadsheet in physics education, e.g.: see [5]-[13]. Spreadsheet has been used to

*Correspondence email address: elisa.founda@gmail.com provide a vivid geometrical picture of lens rays [13]. A simulation of projectile motion has been built in spreadsheet [8]. Trajectories of charged particles also can be visualised using a spreadsheet [12].

Although spreadsheet is widely used in introductory physics course, the use of spreadsheet in advanced topic like fibre optics is still limited. On the other hand, the mathematical equation in fibre optics is more complex. Visualisation with spreadsheet will be helpful for students in interpreting the complex equation and making link between theory and experiment. In this paper, we want to present another spreadsheet application in physics education. We use spreadsheet to visualise the spatial distribution of light intensity on an optical fibre core. The visualisation might be useful for students who take an advanced physics course.

\section{Theory}

Typically, optical fibre is constructed from two main components, i.e. core and cladding, such as illustrated in Figure 1. The refractive index of the cladding must be lower than the core to enable total internal reflection in the core. Total internal reflection is the key mechanism to keep the light stay in the core. It causes the fibre to act as a waveguide.

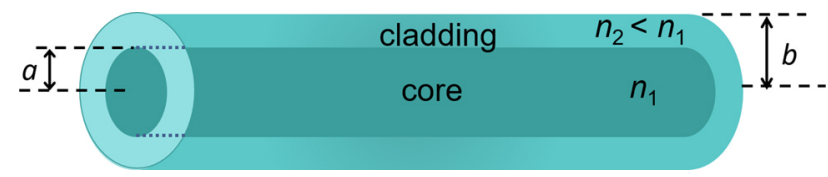

Figure 1: The structure of an optical fibre. 
Light is an electromagnetic wave; it has magnetic and electric field components. The electric field $(\bar{E})$ and magnetic field $\bar{H}$ is perpendicular to each other. Both of them propagate along optical fibre axis as transversal waves. Mathematically, the electric and magmatic field propagation can be expressed as:

$$
\begin{aligned}
& \bar{E}=E_{x}(r, \phi, z) \hat{i}=E_{x 0}(r, \phi) e^{-i \beta z} \hat{i} \\
& \bar{H}=H_{y}(r, \phi, z) \hat{j}=H_{y 0}(r, \phi) e^{-i \beta z} \hat{j}
\end{aligned}
$$

All components of the electric and magnetic fields follow the Helmholtz equation, i.e. $\nabla^{2} U+n^{2}(r) k_{o}^{2} U=0$. In the cylindrical coordinate, the electromagnetic field follows the equation:

$$
\frac{\partial^{2} E_{x}}{\partial r^{2}}+\frac{1}{r} \frac{\partial E_{x}}{\partial r}+\frac{1}{r^{2}} \frac{\partial^{2} E_{x}}{\partial \phi^{2}}+\left(n^{2} k_{0}^{2}-\beta^{2}\right) E_{x}=0
$$

Function of $E_{x 0}(r, \phi)$ in Equation (1) can be written as the product of two functions of $r$ and $\phi$ :

$$
E_{0 x}(r, \phi)=R(r) \Phi(\phi)
$$

We apply Equation (4) and Equation (1) to Equation (3) to get:

$$
\frac{r^{2}}{R} \frac{d^{2} R}{d r^{2}}+\frac{r}{R} \frac{d R}{d r}+r^{2}\left(n^{2} k_{0}^{2}-\beta^{2}\right) R=-\frac{1}{\Phi} \frac{d^{2} \Phi}{d \phi^{2}}
$$

The left-hand side of Equation (5) only depends on $r$, while the right-hand side of Equation (5) only depends on $\phi$. Both sides of Equation (5) must be equal to a constant since $r$ and $\phi$ are varying independently. By defining the constant is $l^{2}$, Equation (5) can be separated as:

$$
\frac{d^{2} \Phi}{d \phi^{2}}+\Phi l^{2}=0
$$

and

$$
\frac{d^{2} R}{d r^{2}}+\frac{1}{r} \frac{d R}{d r}+R\left(n^{2} k_{0}^{2}-\beta^{2}-\frac{l^{2}}{r^{2}}\right)=0 .
$$

The solution of Equation (6) is simply a cosine or sine function. It can be written as:

$$
\Phi(\phi) \propto \cos (l \phi+\alpha),
$$

where $\alpha$ is constant phase shift and $l$ is an integer; $l$ is also known as azimuth number. For optical fibre core, the solution of differential equation in Equation (7) is the Bessel function of the first kind, $J_{l}\left(k_{T} r\right)$.Meanwhile, for the optical fibre cladding, the solution is the modified Bessel function of the second kind, $K_{l}(\gamma r)$ [14]. These solutions may be written as follows:

$$
R(r) \propto\left\{\begin{array}{cr}
J_{l}\left(k_{T} r\right), & r<a \text { (core) } \\
K_{l}(\gamma r), & r \geq a \text { (cladding) }
\end{array}\right.
$$

where $k_{T}^{2}$ and $\gamma^{2}$ are defined as:

$$
k_{T}^{2}=n_{1}^{2} k_{0}^{2}-\beta^{2}
$$

$$
\gamma^{2}=\beta^{2}-n_{2}^{2} k_{0}^{2}
$$

By combining Equation (9) and Equation (8) and by taking the phase shift $\alpha=0$, the full solution of the electric field will be:

$$
E_{x}=\left\{\begin{array}{c}
E_{0} J_{l}\left(k_{T} r\right) \cos l \phi e^{-i \beta z}, \quad r<a \text { (core) } \\
E_{0} K_{l}(\gamma r) \cos l \phi e^{-i \beta z}, \quad r \geq a \text { (cladding) }
\end{array}\right.
$$

Within an analogous way, the full solution of the magnetic field can be expressed as [14]:

$$
H_{y}=\frac{E_{x}}{\eta}
$$

where $\eta$ is a constant called intrinsic impedance.

The scalar function of the field in the Equation (12) must be continuous and have a continuous derivative at $r=a$. Those conditions are satisfied if:

$$
\frac{\left(k_{T} a\right) J_{l}^{\prime}\left(k_{T} a\right)}{J_{l}\left(k_{T} a\right)}=\frac{(\gamma a) K_{l}^{\prime}(\gamma a)}{K_{l}(\gamma a)}
$$

The Bessel functions and their derivatives satisfy these following relations:

$$
\begin{gathered}
J_{l}^{\prime}(x)= \pm J_{l \mp 1}(x) \mp l \frac{J_{l}(x)}{x}, \\
K_{l}^{\prime}(x)=-K_{l \mp 1}(x) \mp l \frac{K_{l}(x)}{x} .
\end{gathered}
$$

By substituting Equation (15) and Equation (16) to Equation (14), we will get the characteristic equation in Equation (17)

$$
X \frac{J_{l \pm 1}(X)}{J_{l}(X)}= \pm Y \frac{K_{l \pm 1}(Y)}{K_{l}(Y)}, \quad Y=\sqrt{V^{2}-X^{2}}
$$

where, $X=k_{T} a, Y=\gamma a$ and $V^{2}=X^{2}+Y^{2}=$ $\left(n_{1}^{2}-n_{2}^{2}\right) k_{0}^{2} a^{2}$

Equation (17) has multiple roots of $X$, even for a fixed $l$; the roots are denoted as $X_{l m}$. Each pair of $l$ and $m$ associated with one mode. A mode is denoted by $\mathrm{LP}_{l m}$. According to Equation (17), the value of $X_{l m}$ are limited by $V$ parameter. The value of $X_{l m}$ can vary between 0 and $V$. Each optical fibre has a specific parameter called fibre parameter or $V$ parameter which governs the number of modes of the fibre and the propagation constant.

For fibre with large $V$ parameters, there are many roots on the interval $0<X<V$. If we let $X$ to be maximum, the right-hand side of Equation (17) will be zero. Hence, Equation (17) can be solved by finding the roots for $J_{l \pm 1}(X)$. The roots of $J_{l \pm 1}(X)$ is denoted as $x_{l m}$ :

$$
\begin{gathered}
x_{l m}=\left(l+2 m-\frac{1}{2} \pm 1\right) \frac{\pi}{2} \approx(l+2 m) \frac{\pi}{2} \\
l=0,1,2, \ldots ; m=1,2, \ldots .
\end{gathered}
$$


Equation (18) determines the cutoff point of mode [15]. Because $X$ should be less than $V$, the allowed modes are restricted by condition:

$$
(l+2 m) \frac{\pi}{2}<V .
$$

Since $X=k_{T} a$, by using Equation 18 , we can determine $k_{T}$.

$$
k_{T} \approx \frac{\pi}{2 a}(l+2 m)
$$

The value of $k_{T}$ depends on $l$ and $m$. Thus, each mode has a specific value of $k_{T}$.

Propagation constant can be determined by substituting Equation (20) to the relation in Equation (10).

$$
\beta \approx \sqrt{n_{1}^{2} k_{0}^{2}-\frac{\pi^{2}}{4 a^{2}}(l+2 m)^{2}}
$$

Propagation constant, $\beta$ should be real to ensure that the specified mode can propagate in the optical fibre. The allowed modes, which satisfy $(l+2 m) \frac{\pi}{2}<V$, always lead to real value of $\beta$.

The intensity of light in optical fibre is equal to the magnitude of poynting vector $\langle\vec{S}>$. Hence [14],

$$
I=|\langle\vec{S}\rangle|=\frac{1}{2} \operatorname{Re}\left\{E_{x} H_{y}^{*}\right\}
$$

The solutions of electric field and magnetic field are substituted to Equation (22) in order to get full expression of light intensity in optical fibre core, $I$.

$$
I=\frac{1}{2 \eta}\left|E_{x}\right|^{2}=\frac{1}{2 \eta} E_{0}^{2} J_{l}^{2}\left(k_{T} r\right) \cos ^{2} l \phi
$$

Or

$$
I=I_{0} J_{l}^{2}\left(k_{T} r\right) \cos ^{2} l \phi
$$

\section{Method}

We utilise spreadsheet software to visualise the spatial intensity distribution on an optical fibre core. The spreadsheet has a surface contour chart feature, which can be used to plot spatial distribution in Equation (23). The polar coordinate is used in Equation (23), but we can only use $x-y$ coordinates in spreadsheet. Thus, we have to transform the polar coordinate to $x-y$ coordinate. This transformation is given as:

$$
r=\sqrt{x^{2}+y^{2}}
$$

and

$$
\phi=\tan ^{-1} \frac{y}{x} .
$$

For visualisation purposes, the radius of the core is fixed to be 0.00007 unit. Thus the $y$ and $x$ coordinates are ranging from -0.00007 to 0.00007 unit. We insert slider using Macros for changing the value of $l$ and $m$ to improve the interactivity of the spreadsheet-based simulation. In this simulation, we assume that the optical fibre has a very large $V$ parameter. The azimuth index, $l$ can be varied from 0 to 100. Meanwhile, the $m$ index can be changed from 1 to 100 . Figure 2 illustrates the spreadsheet. The formula for calculating the spatial distribution is presented in Table 1.

\section{Results and Discussion}

In this study, we construct a simple simulation using spreadsheet to visualise the spatial distribution of light intensity on the optical fibre core for various $l$ and $m$.

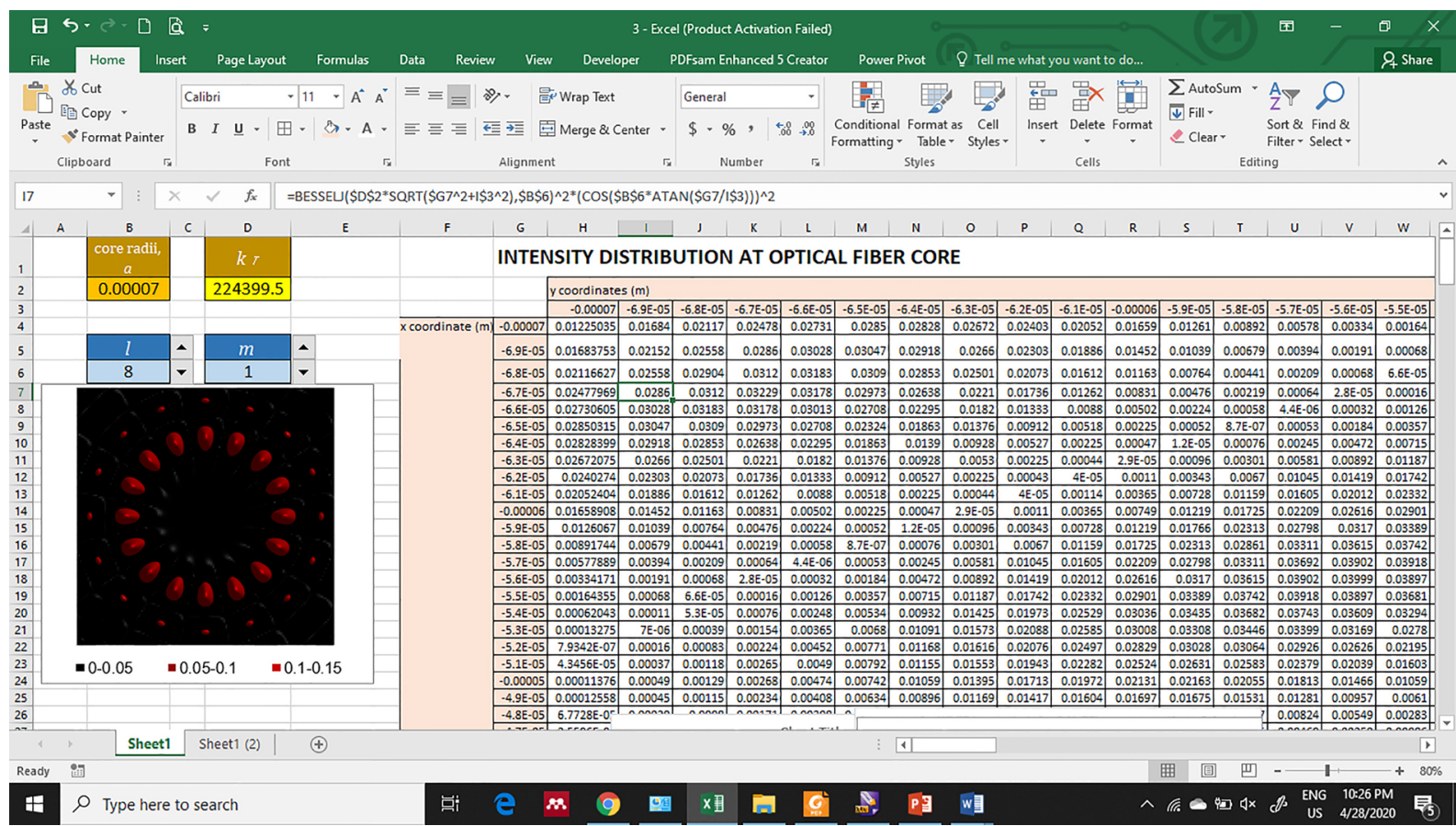

Figure 2: Screenshot of the spreadsheet used to study spatial intensity distribution at the optical fibre core. 
Table 1: The formula used in the spreadsheet

\begin{tabular}{|l|l|l|}
\hline Cell & Variable & Formula \\
\hline B2 & Core radii $a$ & $=0.00007$ \\
B6 & Azimuth index $(l)$, & input range $(1,100)$, interval 1 \\
D2 & $k_{T}$ coefficient & $=\left(\mathrm{B} 6+2^{*} \mathrm{D} 6\right)^{*} \mathrm{PI}() / 2 / \mathrm{B} 2$ \\
D6 & Index $m$ & input range $(1,100)$, interval 1 \\
G3-ER3 & $y$-coordinate & range $(-0.00007,0,000007)$, interval 0.000001 \\
G4-F144 & $x$-coordinate & range $(-0.00007,0,000007)$, interval 0.000001 \\
H4-ER144 & Intensity & $\left.=\mathrm{BESSELJ}\left(\$ \mathrm{D} \$ 2^{*} \mathrm{SQRT}\left(\$ \mathrm{G} 4{ }^{\wedge} 2+\mathrm{H} \$ 3\right\urcorner 2\right), \$ \mathrm{~B} \$ 6\right)^{\wedge} 2^{*}\left(\mathrm{COS}\left(\$ \mathrm{~B} \$ 6^{*} \mathrm{ATAN}(\$ \mathrm{G} 4 / \mathrm{H} \$ 3)\right)\right)^{\wedge} 2$ \\
\hline
\end{tabular}

Figure 3 shows the spatial distribution of intensity on the core of optical fibre for $l=0,1,2,3$, and $m=1,2$. For $l=0$, the intensity peak is located at the central part. There is only one lobe that exists. For $l=1$, the
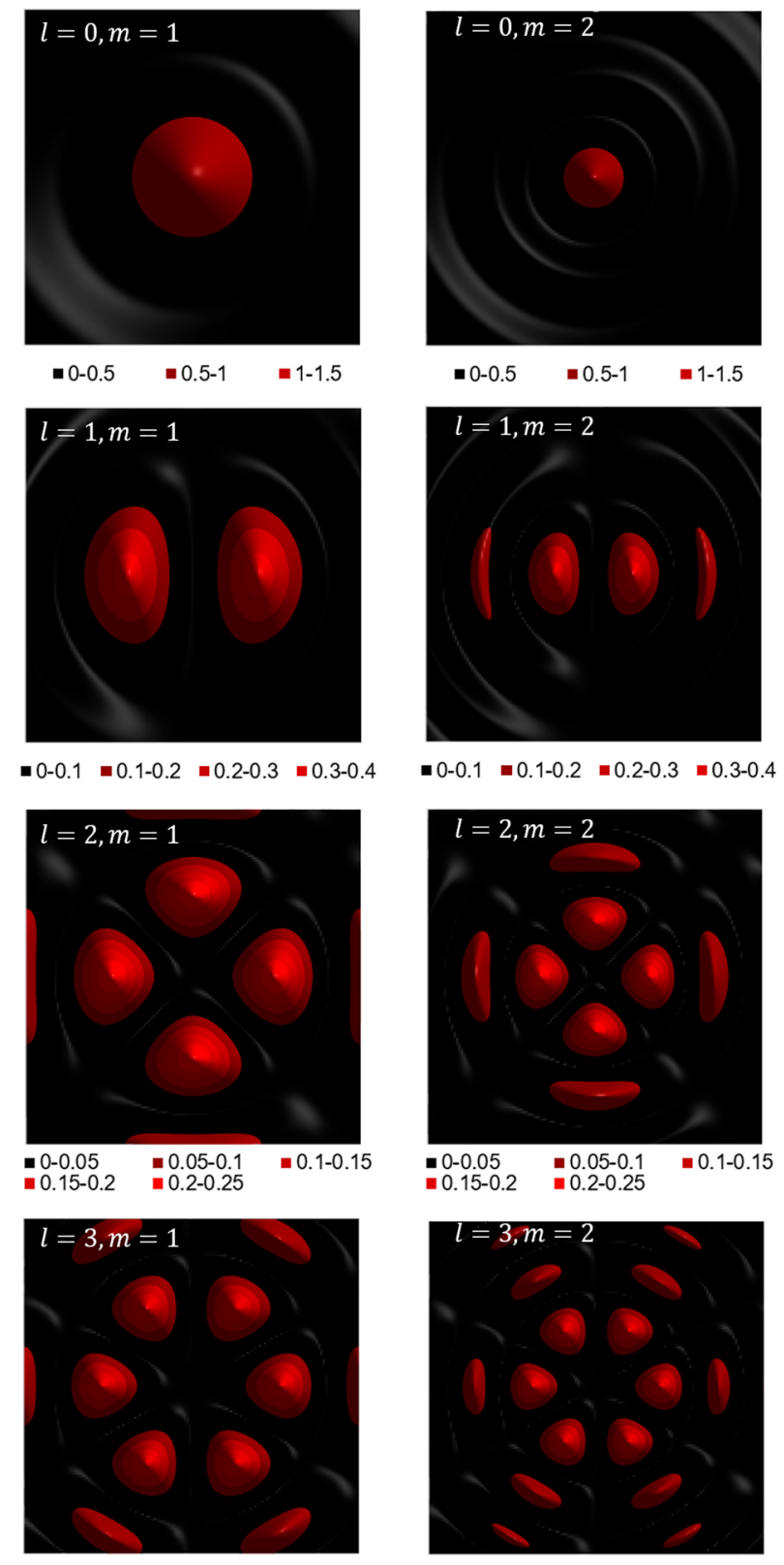

- $0-0.05=0.05-0.1=0.1-0.15=0.15-0.2$

$=0-0.05=0.05-0.1=0.1-0.15=0.15-0.2$

Figure 3: Spatial distribution of intensity on the optical fibre core with $\mathrm{I}=0,1,2,3$, and $\mathrm{m}=1,2$. light distribution at the central part is broken up into two lobes. The intensity is not centralised anymore. For $l=2$, we can see 4 lobes at the centre. For $l>0$, we see that there is a pattern to calculate the number of lobes, $n=2 l$

For $l>0$, there is no light intensity at the central point of the core, forming a hollow dark region. As the value of $l$ becomes higher, the hollow area at the centre becomes wider (see Figure 4). If we look at the intensity distribution for high $l$, the intensity becomes weaker. For $l=25$, the light is almost invisible (see Figure 5).

The spatial intensity patterns visualised in this simulation are in agreement with previous experimental works. Intensity patterns for $l=0, m=1\left(\mathrm{LP}_{01}\right) ; l=1, m=$ $1\left(\mathrm{LP}_{11}\right) ; l=1, m=2\left(\mathrm{LP}_{12}\right)$; and $l=2, m=1\left(\mathrm{LP}_{21}\right)$ in Figure 3 are similar with the experimental result reported in [16]. The intensity pattern for $l=3, m=1$ $\left(\mathrm{LP}_{31}\right)$ resembles the $\mathrm{LP}_{31}$ mode pattern reported in [17]. Spatial Intensity pattern for $l=8, m=1\left(\mathrm{LP}_{81}\right)$ (see Figure 4 ) is in agreement with experimental observation conducted in [18].

Figure 5 illustrates the spatial distribution of intensity on the optical fibre core with high $l$. As can be seen, the intensity is very low for modes with higher $l$. This phenomenon will naturally occur as the field decay significantly as $l$ becomes larger. Equation 22 describes that the intensity is proportional to the square of the field. The field itself is in the form of the Bessel function of the first kind. Bessel function of the first kind (for $l>0$ ) is an oscillating function with decaying amplitude. As the $k_{T}$ and $l$ become larger, the function becomes more oscillating, and the amplitude becomes smaller. Moreover, as $l$ become larger, the first peak of the oscillation tends to move away from the centre of the fibre. It leads to the wider hollow area at the centre of the optical fibre core. The profile of Bessel function and squared Bessel Function for various values of $l$ are given in the Supporting Information.

\section{Strategic Implementation in the Classroom}

This simple simulation can be embedded in a physics classroom when discussing topics about optical fibre modes. The teacher can start from mathematical derivation and then show the simulation to visualise the equa- 


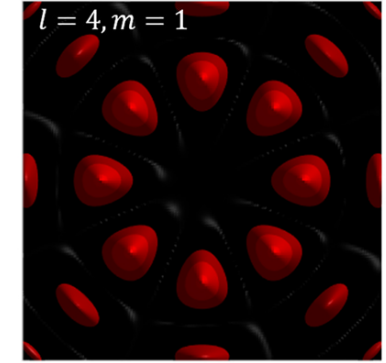

- $0-0.05=0.05-0.1=0.1-0.15=0.15-0.2$

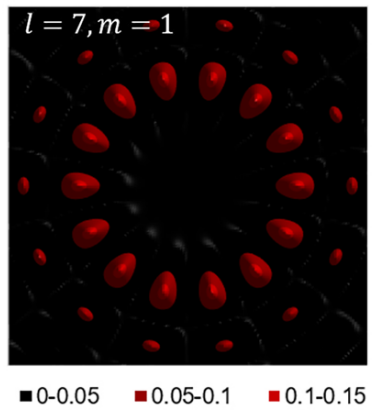

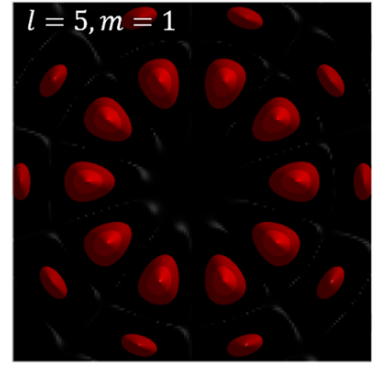

$=0-0.05=0.05-0.1=0.1-0.15$

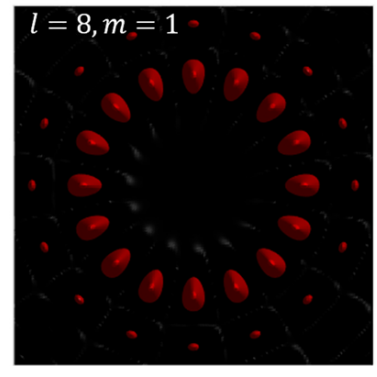

$=0-0.05 \quad=0.05-0.1 \quad=0.1-0.15$
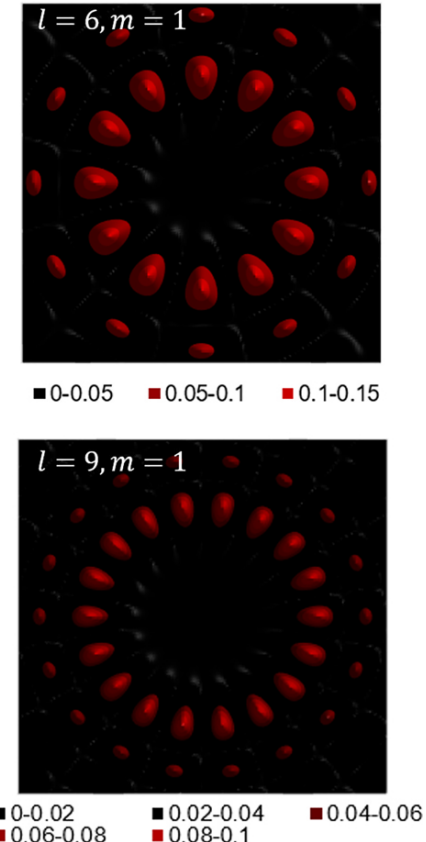

Figure 4: Spatial distribution of intensity on the optical fibre core with $\mathrm{I}=0,1,2,3$, and $\mathrm{m}=1$.
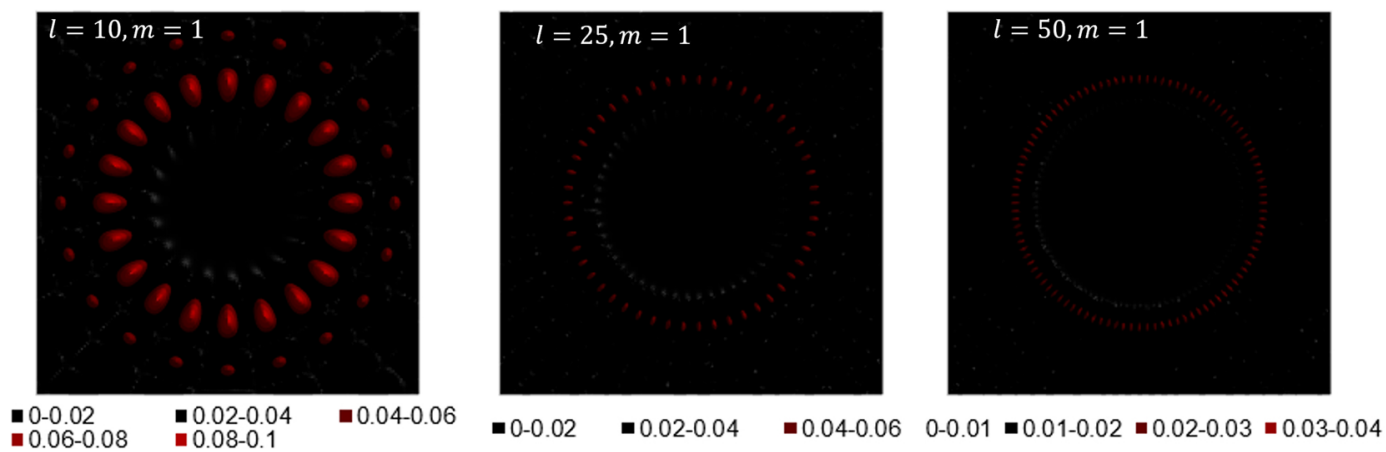

Figure 5: Spatial distribution of intensity on the optical fibre core with $\mathrm{I}=10,25,50$, and $\mathrm{m}=1$.

tion. Another option is students are asked to build their simulation after deriving the mathematical equation.

The simulation can also become a bridge between theoretical modelling and experiment. Students and teachers can experiment with optical fibre modes in the laboratory and then compare their observations with the theoretical modelling-based simulation. When we have to deal with distance learning, in particular during the pandemic period, the experiment can be replaced by a video demonstration. Video demonstration about optical fibre modes are available free in some websites, such as in MIT OpenCourseWare [19]

\section{Conclusions}

In this paper, we have shown one application of spreadsheet software in an advanced physics classroom. We construct spreadsheet-based physics simulation to visualise the spatial intensity distribution on the optical fibre core. More advanced software such as Matlab, Maple or Wolfram Mathematica can also be used to construct the visualisation. Visualisation of core intensity profile in optical fibre is also available free in the Wolfram Demonstration Project [20]. We choose the spreadsheet program simply because many students and teachers are already familiar with it. The simulation helps students to interpret a mathematical equation and understand the physics concept under consideration. Moreover, it can also be a bridge between theoretical modelling and experiment results.

\section{Acknowledgements}

The authors would like to thank Universitas Negeri Yogyakarta and Widya Mandala Catholic University Surabaya for supporting this work.

\section{Supplementary material}

The following online material is available for this article: 
Figure S1. The Plot of Bessel function of the first kind $J_{l}\left(k_{T} r\right)$ versus the distance from the center of optical fiber, $r$ for various order, $l$.

Figure S2. Plot of The Squared-Bessel function of the first kind $J_{l}^{2}\left(k_{T} r\right)$ versus the distance from the center of optical fiber, $r$ for various order, $l$.

\section{References}

[1] L.K. Smetana and R.L. Bell, Int. J. Sci. Educ. 34, 9 (2012).

[2] J.D. Gobert, L. O’Dwyer, P. Horwitz, C.B. Barbara, S.T. Levy and U. Wilensky, Int. J. Sci. Educ. 33, 5 (2011).

[3] M. Develaki, J. Sci. Educ. Technol. 28, 4 (2019).

[4] T. de Jong, M.C. Linn and Z.C. Zacharia, Science 340, $6130(2013)$

[5] Z. Uddin, M. Ahsanuddin and D.A. Khan, Phys. Educ. $\mathbf{5 2 ,} 5$ (2017).

[6] A.L. Subramaniyan, Lat. Am. J. Phys. Educ. 7, 3 (2013).

[7] G. Robinson and Z. Jovanoski, Spreadsheets Educ. 4, 3 (2011).

[8] J. Benacka, Phys. Educ. 50, 1 (2014).

[9] J. Benacka, Spreadsheets Educ. 2, 3 (2008).

[10] G. Singh and K. Siddiqui, J. Educ. Technol. Syst. 37, 4 (2009).

[11] J. Reader, Comput. Phys. 11, 2 (1997).

[12] I. Singh, K.K. Khun and B. Kaur, Phys. Educ. 54, 1 (2019).

[13] M.I. González, Phys. Educ. 53, 3 (2018).

[14] J.A. Buck, Fundamentals of Optical Fibers (John Wiley \& Sons, New York, 2004), v. 2.

[15] C. Kao and P.S.J. Russell, in Fundamental of Photonics, edited by B.E.A. Saleh and M.C. Teich (John Wiley \& Sons, New Jersey, 2007).

[16] H.H. Cerecedo-Núñez, A. Sánchez-Martínez, P. PadillaSosa and G. Rodríguez-Zurita, Rev. Mex. Fis. E 55, 1 (2009).

[17] K. Igarashi, D. Souma, T. Tsuritani and I. Morita, Opt. Express 22, 17 (2014).

[18] J. Carpenter and T.D. Wilkinson, J. Light Wave Technol. 30, 10 (2012).

[19] S. Ezekiel, Multi-mode Fiber, available in: https://ocw.mit.edu/resources/res-6-006-videodemonstrations-in-lasers-and-optics-spring2008/demonstrations-in-physical-optics/multimode-fiber/, accessed 12/06/2020.

[20] J. Miller, Core Intensity Profiles for Linearly Polarised Modes in Optical Fibers, available in: http://demonstrations.wolfram.com/ CoreIntensityProfilesForLinearlyPolarizedModesInOpticalFiber/, accessed 12/06/2020. 